\title{
The structure of plant-herbivore ecological networks varies along elevation gradients
}

\author{
Camille Pitteloud ${ }^{1}$, Jean-Claude Walser $^{2}$, Patrice Descombes ${ }^{3}$, Charles Novaes de \\ Santana ${ }^{1}$, Sergio Rasmann ${ }^{4}$, and Loïc Pellissier ${ }^{5}$ \\ ${ }^{1}$ ETH Zürich \\ ${ }^{2}$ Genetic Diversity Center \\ ${ }^{3}$ University of Fribourg \\ ${ }^{4}$ University of Neuchâtel \\ ${ }^{5} \mathrm{ETH}$ Zurich
}

May 6, 2020

\begin{abstract}
Ecological gradients are expected to be associated with structural rewiring of species interaction networks. The study of network structures along geographic and ecological gradients, however, remains marginal because documenting species interactions at multiple sites is a methodological challenge. Using a standardized DNA metabarcoding method applied to feces, we examined how structural properties of plant-orthoptera networks reflecting specialization and resilience vary with elevation. We found an increase in levels of generality and nestedness with decreasing temperature, and the correlation was stronger than in null models. These relationships corresponded to greater robustness and reduced importance of keystone species in alpine habitats. In cold environments, plant-herbivore networks are wired in a way that may reinforce the resilience of the system to species extinction. Documenting ecological networks along ecological gradients allows a better understanding of the influence of climate on the structure of ecosystems.
\end{abstract}

The structure of plant-herbivore ecological networks varies along elevation gradients

Camille Pitteloud ${ }^{1,2}$, Jean-Claude $W^{2}{ }^{3}{ }^{3}$, Patrice Descombes $^{2}$, Charles Novaes de Santana ${ }^{1,2}$, Sergio Rasmann ${ }^{4}$, Loïc Pellissier ${ }^{1,2}$

\section{Author affiliations}

${ }^{1}$ Landscape Ecology, Institute of Terrestrial Ecosystems, Department of Environmental Systems Science, ETH Zürich, 8092 Zürich, Switzerland

${ }^{2}$ Unit of Land Change Science, Swiss Federal Institute for Forest, Snow and Landscape Research WSL, 8903 Birmensdorf, Switzerland

${ }^{3}$ Genetic Diversity Centre, ETH Zürich, 8092 Zürich, Switzerland

${ }^{4}$ Functional Ecology Laboratory, Institute of Biology, University of Neuchâtel, 2000 Neuchâtel, Switzerland

E-mail addresses camille.pitteloud@usys.ethz.ch; jean-claude.walser@env.ethz.ch; patrice.descombes@wsl.ch; charles.santana@gmail.com; sergio.rasmann@unine.ch; loic.pellissier@usys.ethz.ch

Statement of authorship CP, LP and SR conceived and designed the study. CP, LP and SR wrote the manuscript text with input from all authors. CP, LP and PD contributed to data collection. CP, JCW, CNS and LP analyzed the data. All authors reviewed and contributed to the manuscript. 
Keywords DNA metabarcoding, trophic network, null model, generality, robustness, nestedness, keystone species.

Type of article Ecology Letters

Short running title Elevation and trophic network structure

Counts Abstract: 142 words, main text: 5207 words, number of references: 81, number of figures: 4, number of tables: 1 , number of appendices: 2

Corresponding author Camille Pitteloud, Institute of Terrestrial Ecosystems, Department of Environmental Systems Science, ETH Zürich, Universitätstrasse 16, 8092 Zürich, Switzerland; camille.pitteloud@usys.ethz.ch; +41.44.632.23.45Abstract

Ecological gradients are expected to be associated with structural rewiring of species interaction networks. The study of network structures along geographic and ecological gradients, however, remains marginal because documenting species interactions at multiple sites is a methodological challenge. Using a standardized DNA metabarcoding method applied to feces, we examined how structural properties of plant-orthoptera networks reflecting specialization and resilience vary with elevation. We found an increase in levels of generality and nestedness with decreasing temperature, and the correlation was stronger than in null models. These relationships corresponded to greater robustness and reduced importance of keystone species in alpine habitats. In cold environments, plant-herbivore networks are wired in a way that may reinforce the resilience of the system to species extinction. Documenting ecological networks along ecological gradients allows a better understanding of the influence of climate on the structure of ecosystems.

\section{Introduction}

Species represent the main building blocks of ecosystems and are connected in webs of positive and negative interactions, which shape ecosystem processes and functioning (Thompson et al. 2012). Given the central role of interactions among species for energy and matter flow between ecosystem compartments (Barnes et al. 2018), studying the structure of ecological networks helps us understand how ecosystem functioning might be disrupted by global changes (Petchey et al. 1999; Tylianakis et al. 2008). The wiring among interacting species is hardly random but rather governed by ecological rules (Bascompte 2010; Laigle et al. 2018). The strength of interactions between species may depend on the degree of matching between functional traits, which are shaped through co-evolutionary processes (Rausher 2001; Laigle et al. 2018). In turn, rules of functional matching might be influenced by variation in environmental conditions, such as temperature (Sentis et al. 2014; Gounandet al. 2016), or by climatic stability (Dalsgaard et al.2011). By inducing changes in species composition, ecological gradients can be associated with shifts in species co-occurrence and their ability to form stable links (Welti \& Joern 2015; Pellissier et al.2018). Moreover, shifting environmental conditions might influence interactions among species even when they are steadily co-occurring (Tylianakis \& Morris 2017). As a result, interactions shifts along climatic clines can lead to changes in the structure of networks (Welti \& Joern 2015). Nevertheless, the geographic variation in networks is poorly studied, owing to the difficulty of documenting multiple ecological networks along climatic gradients.

There are major challenges to the large-scale study of ecological networks that relate to the documentation of interactions and the methods used to perform network comparisons at the landscape scale (Pellissier et al. 2018). The study of ecological networks along environmental gradients has so far been limited by the difficulty of observing comparable interactions simultaneously at multiple locations. Novel DNA metabarcoding methods, which are increasingly cheaper, faster and more comprehensive, have opened such opportunities (Kaartinenet al. 2010; Roslin et al. 2019). Deagle et al.(2007) were among the first to develop a DNA metabarcoding protocol to reconstruct the trophic regime of the macaroni penguins on Heard Island in the Indian Ocean. Since then, the study of entire ecological networks has been facilitated through the adaptation of DNA metabarcoding techniques to different sample sources, which enables the collection of many samples over a short period of time (Roslin et al. 2019). For instance, Pornon et al. (2016) developed a protocol to quantify plant-pollinator interactions from pollen samples in the French Central Pyrenees, while Ibanez et 
al. (2013) applied this approach to insect feces for studying the diet of insect herbivores. However, most protocols for network reconstruction were not designed for studies with large spatial scales, and not all were aimed at species-level resolution. In addition, a complete description of the wet lab and bioinformatic procedures is not always accessible, limiting the adaptability and reproducibility of the techniques used to document species interactions. Scaling up the utilization of DNA metabarcoding to entire landscapes, while also sharing methodological workflows as detailed and user-friendly protocols, can spur advances in the study of species interactions along environmental gradients.

From the wide range of natural gradients impacting species distribution and interaction patterns, montane clines represent optimal natural laboratories to understand how species and their interactions vary over environmental gradients (Körner 2003). Changes in climate - most notably temperature - along elevation gradients cause strong environmental filtering in communities (Rahbek 1995; Hodkinson 2005) and can therefore also be expected to influence the structure of ecological networks (O'Connor et al. 2009; Welti \& Joern 2015). The structure of ecological networks along environmental gradients can change as a result of two main processes: (i) a turnover of the species in the network, or (ii) a turnover of the links in the network, in which co-occurring species rewire their interactions along the gradient (Gravel et al. 2019). In particular, the steady decrease in temperature with increasing elevation has been associated with changes in species richness and abundance (Rahbek 1995; Hodkinson 2005; Descombes et al. 2017), likely influencing the networks of species interactions (Adedoja et al. 2018; Pellissier et al. 2018). Therefore, studying changes in the architecture of species networks along elevation gradients contributes to evaluations of the effect of temperature on community structure and stability.

Changes in species' interactions within networks can be summarized by a set of indicators relating to the degree of structuration and complexity of the network (Delmas et al. 2019), including connectance (Martinez 1992), generality (Bersier et al. 2002), nestedness (Bascompte et al. 2003) and robustness (Dunne et al.2002). Metrics of network structure can also quantify the resilience of the networks to environmental disturbances (Thebault \& Fontaine 2010). Specialized networks have been found to be associated with lower robustness against species extinction (Lafferty \& Kuris 2009; Tylianakis \& Morris 2017; but see May 1973; McCann 2000). This is the result of the existence of keystone species (Paine 1969), which are nodes of interactions on which relies the system stability (Poweret al. 1996). The importance and identity of keystone species, but also general structural properties involved in network resilience, may reshuffle along elevation clines. Three main non-exclusive hypotheses have been proposed to support this pattern: (i) at higher elevations the environment is expected to be less predictable (Barry 2008), and survival under these conditions necessitates the evolution of a broader diet breadth (Macarthur \& Levins 1967); (ii) more intense competition at low elevations is predicted to select for more specialized diets to decrease niche overlap (Macarthur \& Levins 1967; Hodkinson 2005); and, more closely linked to plant-herbivore interactions, (iii) a decline in the capacity of plants to resist herbivore attack at higher elevations is expected to facilitate a larger diet breadth of herbivores (Pellissier et al. 2012a; Rasmannet al. 2014; Moreira et al. 2018). In contrast to the expectation of increased herbivore generality at higher elevations, where plant communities are less diverse, it has been proposed that higher plant species richness could benefit insect generalists simply by increasing the availability of species to feed on (Unsicker et al. 2008, Welti et al. 2017).

The comparison of ecological networks along environmental gradients has the inherent methodological difficulties of network comparison (Pellissier et al. 2018). Effective analyses of network structure isolate the influence of real interaction patterns on network structural indices from the effects of network size or sampling design (Banašek-Richter et al. 2004). Studying ecological networks along large-scale environmental clines is challenging in this regard, as the decline in species richness at the extremes of the gradient might result in significant variation in species richness, in turn affecting measures of structural indices sensitive to matrix size (Trøjelsgaard \& Olesen 2016; Pellissier et al. 2018). For instance, the number of links per species inevitably declines from large to small networks, as larger networks include more possible links. Several strategies have been developed to alleviate the confounding effects of comparing networks of different sizes (see Pellissier et al. 2018 for a review of these approaches). The most commonly used approach is the null model, where networks of randomly distributed interactions are generated and compared with the empirical 
patterns. This method has been established to isolate the role of observed interaction patterns on the network structure from the effect of matrix size variation when comparing networks along environmental gradients (Vázquez \& Aizen 2003).

In this study, we investigated the variation in the structure of plant-orthoptera ecological networks along elevation gradients. Orthoptera are among the most abundant herbivorous arthropods in semi-natural grasslands of the European Alpine system, and they strongly impact the functioning of these ecosystems (Blumer \& Diemer 1996). We optimized a protocol for plant DNA metabarcoding applied to orthopteran feces in order to reconstruct plant-orthoptera bipartite networks across 48 study sites situated along six elevation transects in the Swiss Alps. We then applied null models to explore the structural variation in plant-orthoptera bipartite networks and determine if lower levels of network organization and increased robustness are associated with the low temperatures of the alpine environment. Specifically, we proposed the following three hypotheses:

1. Levels of generality in orthoptera should increase in colder environment (higher elevation) according to the following lines of argument: generalist feeders are better equipped to compensate for higher environmental uncertainty; lower interspecific competition attenuates positive selection for specialization; and the reduced plant chemical defences typically found at higher elevations offer more dietary opportunities for insects.

2. The robustness of the network after simulated plant primary extinctions should be higher in colder environments (at high elevations). The increase in insect generalist feeding behavior predicted in the first hypothesis should allow networks of alpine communities to better compensate for possible plant species loss.

3. If more generalist insect herbivores are present and the network is more robust in colder environment, the removal of plant species should induce fewer extinctions within orthopteran communities than at low elevation so that plants have lower keystone weights.

\section{Materials and methods}

\section{Field data collection}

To study variation in the plant-insect trophic network with elevation, we established six elevation transects that covered the diversity of environmental conditions of the Swiss Alps, differing in local climate and bedrock - i.e. in the areas of Bex, Calanda, Faido, Grindelwald, Martigny and Salgesch (Supporting information, Appendix S1, Fig. S1). Each transect was divided into eight sites, spanning elevations from 578 to $2417 \mathrm{~m}$ a.s.l., located on average $240 \mathrm{~m}$ of elevation apart from each other. Sites were chosen to be open grasslands with a limited impact from anthropogenic activities. At each site, we defined a $10 \mathrm{~m} \times 10 \mathrm{~m}$ survey plot representative of the homogeneous composition of the surrounding vegetation. Orthopteran surveys were conducted under sunny weather conditions during the summer at insect peak activity times. We focused on Caelifera and Ensifera suborders that are known to feed on living plant material (Baur et al. 2006). We identified orthoptera by visual inspection, caught on average 10 individuals per species, kept them in falcon tubes for c. $2 \mathrm{~h}$, for collecting fecal excretions, before releasing them again all at once. We performed the vegetation surveys in a $9 \mathrm{~m}^{2}$ circular plot located in the most homogeneous zone of the $100 \mathrm{~m}^{2}$ plot and searched for additional rare species within the $100 \mathrm{~m}^{2}$ plot. We used temperature as the main environmental variable that changed along the elevation gradient (Appendix S1, Fig. S2). Soil temperature data were collected for half of the sites per transect using temperature loggers (DS1921G-F5 HomeChip, Newton Longville, England) that were parameterized at a $0.5^{\circ} \mathrm{C}$ resolution with a sampling rate of 240 minutes, wrapped in parafilm, protected by a silicone capsule and buried $4 \mathrm{~cm}$ deep in the ground at each site (from October 2017 to October 2018). Summer soil temperature was extrapolated for unmonitored sites by linear regression (Appendix S1, Fig. S3). To study the keystone plant species, we measured plant functional traits that related to physical resistance or nutrient content: specific leaf area (SLA), leaf dry matter content (LDMC), force required to pierce the leaf lamina (punch), and carbon-to-nitrogen ratio $(\mathrm{C} / \mathrm{N})$. We sampled well-developed, healthy leaves to measure the traits of all species with a minimum of three replicates across their elevation range $(76 \%$ of the total number of surveyed species). SLA and LDMC were measured using standard 
procedures (Pérez-Harguindeguy et al. 2013). Punch was calculated using a digital force gauge (IMADA CO., LTD. Toyohashi, Japan), following Sanson et al. (2001). C/N was determined by dry combustion of ground leaf material $(4 \mathrm{mg}+/-0.2 \mathrm{mg})$ of intraspecific replicates pooled to equal weight using an elemental analyzer (NC-2500 from CE Instruments, Wigan, Lancashire, United Kingdom). The collection of trait data was completed with published datasets (Kattge et al. 2011; Körner et al. 2016; Descombes et al. 2017).

\section{Plant-orthoptera network reconstruction}

The reconstruction of plant-insect trophic networks from fecal samples relies on a DNA metabarcoding procedure that uses a two-step DNA amplification PCR-based approach in which samples are individually tagged by dual-indexing. A full protocol of the wet-lab procedure, from DNA extraction to sequencing, is provided in Supporting information, Appendix S2, section Methods 1. In short, after DNA extraction from the insect feces, the ITS2 nuclear plant marker (360bp) was amplified in the amplicon PCR. We selected this marker based on its ease of amplification, high taxonomic resolution, good coverage of the reference database and successful application to degraded DNA samples (Li et al. 2011; García-Robledo et al. 2013). In parallel to DNA metabarcoding library preparation and sequencing, we compiled an ITS2 reference database by recovering sequences from Genbank (Clark et al. 2016). The database was filtered using in-silico PCRs that allow only one mismatch between the primers and the priming sites. We expanded the reference database with custom sequences generated for $54 \%$ of the plant species (see Appendix S2, section Methods 2). After processing of the raw sequencing data, OTU calling and taxonomic assignment against the DNA reference database, the OTU table was streamlined to reconstruct individual networks using R (R Core Team 2019; see complete descriptions of the bioinformatic and OTU table cleaning procedures in Appendix S2, section Methods 3). We discarded OTUs that were non-monophyletic and/or identified above the family level, summed the OTUs belonging to the same taxon, used vegetation surveys to filter out the OTUs with low read numbers, and redistributed the count of OTUs assigned above the species level. In a final step, we computed the relative read abundance (RRA) for each sample as an estimate of the interaction intensity (Deagleet al. 2019, Roslin et al. 2019).

\section{Statistical analyses of variation in network metrics}

We computed network metrics using the $\mathrm{R}$ package bipartite(Dormann et al. 2008). We considered the number of links per species, calculated as the total number of links divided by the total number of species. The generality index, which estimates the mean number of plant species per orthopteran species weighted by the marginal counts (Bersier et al. 2002) and is calculated from presences and absences of interactions instead of their intensities. The robustness of the networks (Dunne et al. 2002), which involves calculating the cumulative proportion of secondary extinctions caused by the sequential removal of plant species until all insect species are extinct. As implemented in bipartite, the function uses a quantitative estimation of the robustness introduced by Burgos et al. (2007). It measures the area under the attack tolerance curve (ATC), which describes the relationship between the proportion of species removed and the proportion of surviving insect species, until all species are extinct. The sequential species removal was done randomly for 100 replicates, excluding plant taxa that were not ingested. We finally computed the weighted nestedness following Galeano et al. (2009), as it has been associated to network robustness (Bascompte et al.2003). Relationships between mean summer temperature and the observed network metrics were tested using linear mixed effects models including transect identity as a random factor (packages lme4 Bateset al. 2008 and lmerTest Kuznetsova et al. 2017). We used a null model approach to discriminate the effect of the nonrandom interactions on the metric from the influence of inherent bias of network metric calculation (e.g. network size). We generated 999 random metawebs, where interactions were fully randomized and impossible links excluded. Individual random networks were then reconstructed for each study site according to their species composition. We further measured network properties for each network and metric variation along the gradient, following the same procedure as applied for the observed networks. Statistical significance of the metric variation was confirmed if the observed slope of the relationship between the temperature and the network metric fell outside the 2.5-97.5\% quantile interval of the slopes obtained for the randomized network of interactions. We also calculated the standardized effect size (SES) to quantify the difference 
between the observed relationships and the null models. The approach we used here does not suppress the metrics' sensitivity to sampling effects, but slope values outside the $2.5-97.5 \%$ quantile interval of the slopes obtained from random networks (and large values of SES) indicate that the interactions of empirical networks contribute more to the metric variation along the gradient than expected by chance.

\section{Identification of keystone species}

Keystone species are defined here as plant taxa that play a major role in providing a food source for the orthopteran herbivores (Millset al. 1993; Power et al. 1996). We identified keystone species using custom R scripts, submitting each network, preliminarily transformed into an igraph object (Csardi \& Nepusz 2006), to a sequential and random removal of plant species. Insect species were considered to be extinct upon loss of all the plant species they feed on. Plant species were removed until all insect species became extinct. This was repeated for $n^{*}(n-1)$ simulations, with $n$ equaling the total number of plant taxa in the network. The mean number of secondary extinctions caused by plant removal, to which we refer hereafter as the keystone score, was then calculated for each plant species. To examine the distribution and the keystone score of species within the plant functional space at the low $(<1050 \mathrm{~m}$ a.s.l. $)$ and high elevation $(>2000 \mathrm{~m}$ a.s.l.), we performed a principal components analysis on plant traits with the function dudi.pca from the ade4 package (Thioulouse et al. 2018). We compared the distribution of species with different keystone scores in the functional space of plant traits of species at the lowest and highest elevation sites. For both elevation classes, we extracted plant species based on their presence within the corresponding elevation range. We further determined the 10 species with the highest keystone scores for each network, averaged their weights for each elevation class and placed them in the functional space of plant trait.

\section{Results}

\section{Field surveys and network reconstruction}

We identified 45 orthopteran species, including 29 and 16 species of the Caelifera and Ensifera suborders, respectively, and we collected 403 feces samples. Vegetation surveys led to the identification of 496 plant species, belonging to 265 genera and 63 families. The DNA barcoding reference database compiled 5969 reference sequences covering the taxonomic diversity of the vegetation surveys for $95.2 \%$ of the families, $92.2 \%$ of the genera and $88.5 \%$ of the species, with $50 \%$ of the missing species having their genus represented in the database. The MiSeq v3 2x300 PE sequencing run provided $31 \mathrm{M}$ reads, which decreased to 15.5 M after filtering, paired-end merging and trimming steps (https://github.com/lh3/seqtk; Magoc \& Salzberg 2011; Martin 2011), with an average sequencing depth of $>36,500$ reads, and to 1774 OTUs after OTU calling (Edgar 2016). Following taxonomic identification that used a stringent threshold of assignment of 0.95, we discarded 105 OTUs that were not monophyletic and 176 OTUs with a taxonomic assignment higher than the family level. After the merging of OTUs belonging to the same taxon, the equal redistribution of read counts to lower taxonomic ranks (Methods and Results sections in Appendix S2) and the addition of species that had not been consumed, the OTU table comprised 601 taxa, including 496 species, 99 genera and 6 families corresponding to taxa that were not identified to a lower taxonomic level in the field. The total number of interactions recorded was 10,615 out of 28,127 possible links. The reconstruction of individual networks exemplified for low- and high-elevation sites of three transects are illustrated in Fig. 1.

\section{Variation in network metrics}

In agreement with our first hypothesis, we found that the generality of the observed networks decreased with increasing temperature (observed slope $=-0.4973, P=0.035$; Fig. 2, Table 1 ) which was consistent with a decrease of the overall network specialization (H2') along the elevation (Appendix S1, Fig. S4a, Table S1). The variation in these metrics differed significantly from null models, as we found their observed slopes to be outside the 2.5-97.5\% quantile interval of the slopes obtained from random networks and SES values were high (generality: $2.5-97.5 \%$ quantile interval $=(-0.4066,-0.1868), \mathrm{SES}=-3.44$; overall network specialization in Table S1). We further found a negative relationship between the weighted nestedness and the temperature for empirical webs that was different from null models (observed slope $=-0.0102, P=$ $0.042,2.5-97.5 \%$ quantile interval $=(-0.0054,-0.0060), \mathrm{SES}=-3.51$; Fig. 2, Table $\mathrm{S})$. We found a positive 
relationship between robustness and temperature (slope $=0.0033, P=0.023$ ), but the observed decrease in robustness in cold environments was lower than expected from nulls models $(2.5-97.5 \%$ quantile interval $=$ $(0.0057,0.0082)$, SES $=-5.77)$, indicating a role of the wiring of interactions in attenuating the decrease in robustness toward colder conditions (Fig. 2). Variation in the number of links per species (Fig. 2, Table 1), the connectance and the trophic niche overlap of orthoptera (Appendix S1, Fig. S4b and S4c, Table S1) were not different between the observed and the randomized networks. We found a positive relationship between temperature and the number of links per species in empirical networks (Fig. 2a, Table 1) but not for the connectance or the niche overlap (Appendix S1, Fig. S4b and S4c, Table S1).

\section{Keystones species in the functional space of plant traits}

We found higher keystone plant species scores in warmer environments (i.e. 0.41 on average for study sites located $<1050 \mathrm{~m}$; Appendix S1, Table S2) than in colder environments (i.e. 0.26 on average for sites located $>2000$ m; Appendix S1, Table S2). The keystone scores were generally low, with the removal of a single plant species resulting, on average, in less than one insect secondary extinction (Appendix S1, Table S2). The top 10 keystones species retrieved from alpine and lowland trophic networks had same dietary preferences for botanical groups, as illustrated by their similar distribution patterns within the functional space of the plant traits (Fig. 3). In both warm and cold environments, the top 10 keystone species occupied the functional space characterized by high punch values (to the maximal extent of this axis; Fig. 3). The top three keystone species of warm and cold environments all belonged to the Poaceae family (Appendix S1, Table S2). Forb keystone species were located in the functional space along the opposing axes of C/N, LDMC vs . SLA in both low- and high-elevation networks; while legume keystone species were mostly distributed along the SLA trait axis, at high but not at low elevation (Fig. 3).

\section{Discussion}

The development of novel high-throughput molecular methods has paved the way for a better understanding of how environmental factors shape the structure of species interaction networks (Nielsen et al. 2018; Roslin et al. 2019). Using an improved and non-invasive metabarcoding procedure based on insect feces, we reconstructed the structure of plant-orthoptera networks across multiple sites along elevation gradients, thus favoring technical advances in the field of 'landscape network ecology'. We showed that networks exhibited structural variation along the ecological gradients, as a result of both the rewiring of species interactions and shifts in network size. Networks of high-elevation cold environments displayed reduced levels of specialization and increased nestedness, which are metrics theoretically expected to be central components of network stability (Fig. 2, Fig. 4; Table 1; Appendix S1, Fig. S4a, Table S1). We argue that when increased, these structural properties confer higher network resilience, presumably through a more homogeneous distribution of the herbivore interaction over the available plant species functional space. Theoretical work on the structure-stability relationship of ecological networks suggests a positive association between network resilience to species extinction and structural indices, including connectance and nestedness (Dunneet al. 2002; Memmott et al. 2004; Lafferty \& Kuris 2009). Our empirical analyses along several elevation transects support theoretical expectations showing that networks in cold environments are less specialized and more nested, which presumably enhance network robustness (Burgos et al. 2007; Miller-Struttmann \& Galen 2014). Novel molecular methods enabling the monitoring of network variation in space, as done in our study, but also in time, should provide new perspectives for understanding the trophic architecture of species assemblages.

The observed higher generality for alpine plant-orthoptera networks agrees with three underlying arguments supporting biotic and abiotic shifts along elevation gradients: (i) lower environmental predictability, (ii) less species competition for resources, and (iii) relaxation of plant chemical defences (Macarthur \& Levins 1967; Hodkinson 2005; Rasmann et al. 2014). First, greater environmental stresses and variation in the alpine belt (Körner 2003; Barry 2008) may impose constraints for insects to complete developmental and reproduction cycles (Hodkinson 2005). In particular, environmental fluctuation at high elevations may increase resource stochasticity, which translates into greater spatio-temporal variation of the host plants than at low elevations (Billings \& Mooney 1968). Cooler and more variable temperatures might also reduce search and digestive 
efficiency in ectothermic animals (Hodkinson 2005). In turn, such environmental unpredictably could be offset through the reinforcement of generalist feeding behavior (Macarthur \& Levins 1967). Overall, orthoptera are generalist feeders (the median number of host plants in our study was 26). Hence, while food plant fluctuation should largely impact the evolutionary specialization of more specialized clades, such as the butterflies (median of eight host plants, Pellissier et al.2012b), orthoptera might more easily compensate for the demographic fluctuations of food plant species by maintaining a large diet breadth (Cates 1981) and other factors could contribute to the observed increase in generality. Second, higher species richness of orthoptera at low elevations (Appendix S1, Fig. S5) might pressure species to escape competition by focusing on distinct and more specialized diets (Macarthur \& Levins 1967; Hodkinson 2005). However, we found no relationship between the species richness of orthoptera and the overall network specialization (Appendix S1, Fig. S6) and species niche overlap among orthoptera did not vary along the temperature gradient, indicating that interspecific competition for plant resources is weakened in orthoptera (Appendix S1, Fig. S4c, Table S1). Third, it was previously shown that alpine plant communities are less resistant to herbivores than lowelevation plant communities (Rasmann et al. 2014; Callis-Duehl et al. 2017). These plant defence patterns could promote a stronger generalist feeding behavior in colder environments, through easier digestibility of various plant materials (Moreiraet al. 2018). Our results indicating lower selectiveness of orthoptera for alpine plants are in agreement with a generalized reduction in defence levels in plants growing at high elevations (Rasmann et al. 2014). We documented that orthopteran communities from cold environments feed on a broader range of plant families and target more intensively families such as Apiaceae, Boraginaceae, Caryophyllaceae and Fabaceae, compared with the feeding habits of lower-elevation orthoptera (Appendix S1, Fig. S7). In particular, we found that some species at higher elevation were extreme generalist, feeding on almost all plant species in the communities (Fig. 4). The presence of those hyper-generalists might contribute to the reported increase of nestedness with elevation (Fig. 2c, Table 1), where, more specialist species interact more often with a proper subset of the most generalist species (Bascompte et al. 2003; Miller-Struttmann \& Galen 2014). Russo et al. (2019) also showed higher nestedness as a result of a prevalence of super-generalist feeders and resources. Altogether, higher generality at the network and species level should increase the robustness of networks to extinctions.

The increase in generality and nestedness of networks at higher elevation (lower temperatures) were associated with an increase in network robustness in cold environments (Fig. 2, Table 1). These results support previously documented co-variation between network robustness and generality (Welti et al. 2017) or nestedness (Araújo 2016) in plant-herbivores systems, and a negative association between temperature and network robustness (Welti et al. 2019). The association between network specialization, nestedness and robustness metrics with the underlying temperature gradient suggests that ecological or evolutionary factors have led to more robust networks in more stressful environments. Consistently, increased generality and nestedness at high elevation may results from a greater number of plants species involved in realized interactions thus decreasing the weight of the keystones species (Fig. 3; Appendix S1, Table S2). In general, orthoptera feed on multiple plant species, so the loss of one plant species is never sufficient to cause the loss of one species of orthoptera (averaged keystone score $<1$ ), but they still show some degree of preference as regard to the functional traits of the plant they are feeding on. In our study, orthoptera showed a preference for plants with tougher leaves (Fig. 3) which typically correspond to monocotyledons (Fig. 1; Appendix 1, Fig. S8, Table S2), some of which were particularly dominant in the studied grasslands (e.g. Bromus, Festuca and Nardus ), as these herbivores are equipped with enough mandibular strength to cut through such leaves (Ibanez et al. 2013a). We found lower and more even keystone scores for alpine plant species, meaning that the removal of plant species at higher elevations was associated with lower secondary extinctions (Table 3). The decrease in the keystone score of grasses at higher sites might also be associated with the decline in the cover of grass vegetation with increasing elevation (Appendix S1, Fig. S9). At high-elevation sites, keystone species also had other functional attributes, including higher SLA but lower $\mathrm{C} / \mathrm{N}$ values (Fig. 3) compared with low-elevation plants, corresponding to more palatable and resource-rich host plants (Pérez-Harguindeguy et al. 2013), providing herbivores with higher nutritive content during the short growing season of the alpine environment. These results suggest that the identity of the keystone species in plant-orthoptera bipartite systems is determined by a combination of factors involving plant species abundances and co-evolutionary 
mechanisms between insect feeding ability and plant defence, presumably resulting from mechanical and chemical defence tradeoffs.

Compared with traditional methods based on visual analyses of feces or gut content or literature-based documentation of interactions (Nielsenet al. 2018), the DNA metabarcoding procedure represents an effective and easily adaptable method for documenting interactions involving plants and insect species. As a compromise between the spatial coverage of our study and the available sampling resources, potential impacts of sampling replication and seasonality and year-to-year change on diet composition were not assessed here (Mata et al. 2019). Overall, our approach may open fields of investigation on the possible spatio-temporal variation in plant-insect interactions by expanding the means for collecting species interaction data.

Taken together, our results show an increase in plant-herbivore network generality and nestedness with elevation, which drives variation in network robustness along the gradient and ultimately gives lower weights to keystone species in alpine than in lowland environments. Shifts in abiotic components can alter the structuring of species interactions directly or indirectly (Welti \& Joern 2015; Tylianakis \& Morris 2017), by influencing the different aspects of the species interface through both abiotic and biotic pressures. We suggest that the observed patterns of network structural variation regarding elevation represent entangled responses of networks to environmental predictability and plant chemical defences, although further investigation would be required to confirm this possibility. Generally, orthoptera are not very sensitive to extinction, in that the loss of multiple plant species is necessary to cause secondary extinctions. Nevertheless, intensification of land use practices in lower-elevation mountain grasslands, for instance through the use of fertilizers, can regularly cause the loss of multiple plant species, which could then lead to extinctions in orthoptera (Chistéet al. 2016). Our study helps paving the way to a better understanding of the eco-evolutionary factors underlying network structure along large-scale ecological gradients, but also highlights how resilient species assemblages are to the accelerated rate of species extinction.

\section{Conflict of interest}

The authors declare that they have no conflict of interest.

\section{Acknowledgements}

We are deeply grateful to our collaborators at the Genetic Diversity Centre (GDC) at ETH Zurich, in particular to Silvia Kobel and Aria Minder for their assistance in producing and analyzing the data used in this paper. A special thanks to Christian Roesti and Maude Poirier for their invaluable help in field sampling. For their assistance in data collection, we thank Giulia Donati, Fabian Fopp, Joan Casanelles Abella, MarieAngèle Pitteloud, Nico Baetz, Nadine Sandau. Thanks also to Camille Albouy, Gabriel Dettling, Daniele Pezzotta, Marco Lanfranchi, Janka Bollenbach, Angéline Bedolla, Alain Reymond, Jean-Nicolas Pradervand, Glenn Litsios, Antoine Guisan and Nicolas Salamin. This work was funded by the National Swiss Fund for research (grants 162604 to LP, 31003A_179481 to SR, 31003A-125145 to Antoine Guisan).

\section{References}

Adedoja, O.A., Kehinde, T. \& Samways, M.J. (2018). Insect-flower interaction networks vary among endemic pollinator taxa over an elevation gradient. PLoS One , 13, e0207453.

Araújo, W.S. de. (2016). Global patterns in the structure and robustness of plant-herbivore networks. Front. Biogeogr., 8.

Banašek-Richter, C., Cattin, M.F. \& Bersier, L.F. (2004). Sampling effects and the robustness of quantitative and qualitative food-web descriptors. J. Theor. Biol. , 226, 23-32.

Barnes, A.D., Jochum, M., Lefcheck, J.S., Eisenhauer, N., Scherber, C., O'Connor, M.I., et al. (2018). Energy flux: The link between multitrophic biodiversity and ecosystem functioning. Trends Ecol. Evol., 33, 186-197. 
Barry, R.G. (2008). Mountain weather and climate . Mt. Weather Clim. 3rd edn. Cambridge University Press, Cambridge.

Bascompte, J. (2010). Structure and dynamics of ecological networks.Science , 329, 765-6.

Bascompte, J., Jordano, P., Melián, C.J. \& Olesen, J.M. (2003). The nested assembly of plant-animal mutualistic networks. Proc. Natl. Acad. Sci. U. S. A. , 100, 9383-9387.

Bates, D., Mächler, M., Bolker, B. \& Walker, S. (2008). Fitting mixed-effects models using the lme4 Package in R. J. Stat. Softw. , 67.

Baur, B., Baur, H., Roesti, C. \& Roesti, D. (2006). Sauterelles, Grillons et Criquets de Suisse . Haupt, Bern.

Bersier, L.-F., Banašek-Richter, C. \& Cattin, M.-F. (2002). Quantitative descriptors of food-web matrices. Ecology , 83, 2394-2407.

Billings, W.D. \& Mooney, H.A. (1968). The ecology of arctic and alpine plants. Biol. Rev. , 43, 481-529.

Blumer, P. \& Diemer, M. (1996). The occurrence and consequences of grasshopper herbivory in an alpine grassland, Swiss central Alps.Arct. Alp. Res. , 28, 435.

Burgos, E., Ceva, H., Perazzo, R.P.J., Devoto, M., Medan, D., Zimmermann, M., et al. (2007). Why nestedness in mutualistic networks? J. Theor. Biol. , 249, 307-313.

Callis-Duehl, K., Vittoz, P., Defossez, E. \& Rasmann, S. (2017). Community-level relaxation of plant defenses against herbivores at high elevation. Plant Ecol. , 218, 291-304.

Cates, R.G. (1981). Host plant predictability and the feeding patterns of monophagous, oligophagous, and polyphagous insect herbivores. Oecologia , 48, 319-326.

Chisté, M.N., Mody, K., Gossner, M.M., Simons, N.K., Köhler, G., Weisser, W.W., et al. (2016). Losers, winners, and opportunists: How grassland land-use intensity affects orthopteran communities.Ecosphere , 7, e01545.

Clark, K., Karsch-mizrachi, I., Lipman, D.J., Ostell, J. \& Sayers, E.W. (2016). GenBank. Nucleic Acids Res. , 44, 67-72.

Csardi, G. \& Nepusz, T. (2006). The igraph software package for complex network research. InterJournal Complex Syst. , 1695, 1-9.

Dalsgaard, B., Magård, E., Fjeldså, J., Martín González, A.M., Rahbek, C., Olesen, J.M., et al. (2011). Specialization in plant-hummingbird networks is associated with species richness, contemporary precipitation and quaternary climate-change velocity.PLoS One, 6, e25891.

Deagle, B.E., Gales, N.J., Evans, K., Jarman, S.N., Robinson, S., Trebilco, R., et al. (2007). Studying seabird diet through genetic analysis of faeces: A case study on macaroni penguins (Eudyptes chrysolophus). PLoS One, 2, e831.

Deagle, B.E., Thomas, A.C., McInnes, J.C., Clarke, L.J., Vesterinen, E.J., Clare, E.L., et al. (2019). Counting with DNA in metabarcoding studies: How should we convert sequence reads to dietary data? Mol. Ecol. , 28, 391-406.

Delmas, E., Besson, M., Brice, M.-H., Burkle, L.A., Dalla Riva, G. V., Fortin, M.-J., et al. (2019). Analysing ecological networks of species interactions. Biol. Rev. , 94, 16-36.

Descombes, P., Marchon, J., Pradervand, Jn.N., Bilat, J., Guisan, A., Rasmann, S., et al. (2017). Communitylevel plant palatability increases with elevation as insect herbivore abundance declines. J. Ecol. , 105, 142-151.

Dormann, C., Gruber, B. \& Fründ, J. (2008). Introducing the bipartite package: analysing ecological networks. $R$ news , 8, 8-11. 
Dunne, J.A., Williams, R.J. \& Martinez, N.D. (2002). Network structure and biodiversity loss in food webs: robustness increases with connectance. Ecol. Lett. , 5, 558-567.

Edgar, R.C. (2016). UNOISE2: improved error-correction for Illumina 16S and ITS amplicon sequencing. bioRxiv, 81257 .

Galeano, J., Pastor, J.M. \& Iriondo, J.M. (2009). Weighted-interaction nestedness estimator (WINE): a new estimator to calculate over frequency matrices. Environ. Model. Softw. , 24, 1342-1346.

García-Robledo, C., Erickson, D.L., Staines, C.L., Erwin, T.L. \& Kress, W.J. (2013). Tropical plant-herbivore networks: reconstructing species interactions using DNA barcodes. PLoS One, 8.

Gounand, I., Kéfi, S., Mouquet, N. \& Gravel, D. (2016). Trait selection during food web assembly: the roles of interactions and temperature. Theor. Ecol. , 9, 417-429.

Gravel, D., Baiser, B., Dunne, J.A., Kopelke, J.-P., Martinez, N.D., Nyman, T., et al. (2019). Bringing Elton and Grinnell together: a quantitative framework to represent the biogeography of ecological interaction networks. Ecography (Cop.). , 42, 401-415.

Hodkinson, I.D. (2005). Terrestrial insects along elevation gradients: species and community responses to altitude. Biol. Rev. , 80, 489.

Ibanez, S., Lavorel, S., Puijalon, S. \& Moretti, M. (2013a). Herbivory mediated by coupling between biomechanical traits of plants and grasshoppers. Funct. Ecol. , 27, 479-489.

Ibanez, S., Manneville, O., Miquel, C., Taberlet, P., Valentini, A., Aubert, S., et al. (2013b). Plant functional traits reveal the relative contribution of habitat and food preferences to the diet of grasshoppers. Oecologia , 173, 1459-1470.

Kaartinen, R., Stone, G.N., Hearn, J., Lohse, K. \& Roslin, T. (2010). Revealing secret liaisons: DNA barcoding changes our understanding of food webs. Ecol. Entomol. , 35, 623-638.

Kattge, J., Díaz, S., Lavorel, S., Prentice, I.C., Leadley, P., Bönisch, G., et al. (2011). TRY - a global database of plant traits. Glob. Chang. Biol. , 17, 2905-2935.

Körner, C. (2003). The alpine plant life: functional plant ecology of high mountain ecosystems . 2nd edn. Springer, Berlin.

Körner, C., Leuzinger, S., Riedl, S., Siegwolf, R.T. \& Streule, L. (2016). Carbon and nitrogen stable isotope signals for an entire alpine flora, based on herbarium samples. Alp. Bot. , 126, 153-166.

Kuznetsova, A., Brockhoff, P.B. \& Christensen, R.H.B. (2017). lmerTest package: tests in linear mixed effects models. J. Stat. Softw. , 83.

Lafferty, K.D. \& Kuris, A.M. (2009). Parasites reduce food web robustness because they are sensitive to secondary extinction as illustrated by an invasive estuarine snail. Philos. Trans. R. Soc. B Biol. Sci., 364, 1659-1663.

Laigle, I., Aubin, I., Digel, C., Brose, U., Boulangeat, I. \& Gravel, D. (2018). Species traits as drivers of food web structure.Oikos , 127, 316-326.

Li, D.-Z., Gao, L.-M., Li, H.-T., Wang, H., Ge, X.-J., Liu, J.-Q., et al. (2011). Comparative analysis of a large dataset indicates that internal transcribed spacer (ITS) should be incorporated into the core barcode for seed plants. Proc. Natl. Acad. Sci. U. S. A. , 108, 19641-6.

Macarthur, R. \& Levins, R. (1967). The limiting similarity, convergence, and divergence of coexisting species. Am. Nat. , 101, 377-385.

Magoc, T. \& Salzberg, S.L. (2011). FLASH: fast length adjustment of short reads to improve genome assemblies. Bioinformatics , 27, 2957-2963. 
Martin, M. (2011). Cutadapt removes adapter sequences from high-throughput sequencing reads. EMBnet.journal , 17, 10.

Martinez, N.D. (1992). Constant connectance in community food webs.Am. Nat. , 139, 1208-1218.

Mata, V.A., Rebelo, H., Amorim, F., McCracken, G.F., Jarman, S. \& Beja, P. (2019). How much is enough? Effects of technical and biological replication on metabarcoding dietary analysis. Mol. Ecol. , 28, 165-175.

May, R.M. (1973). Stability and complexity in model ecosystems.

Monogr. Popul. Biol.

McCann, K.S. (2000). The diversity-stability debate. Nature , 405, 228-233.

Memmott, J., Waser, N.M. \& Price, M. V. (2004). Tolerance of pollination networks to species extinctions. Proc. R. Soc. B Biol. Sci. , 271, 2605-2611.

Miller-Struttmann, N.E. \& Galen, C. (2014). High-altitude multi-taskers: bumble bee food plant use broadens along an altitudinal productivity gradient. Oecologia , 176, 1033-1045.

Mills, L.S., Soulé, M.E. \& Doak, D.F. (1993). The Keystone-Species Concept in Ecology and Conservation. Bioscience, 43, 219-224.

Moreira, X., Petry, W.K., Mooney, K.A., Rasmann, S. \& Abdala-Roberts, L. (2018). Elevational gradients in plant defences and insect herbivory: recent advances in the field and prospects for future research.Ecography (Cop.). , 41, 1485-1496.

Nielsen, J.M., Clare, E.L., Hayden, B., Brett, M.T. \& Kratina, P. (2018). Diet tracing in ecology: Method comparison and selection. Methods Ecol. Evol. , 9, 278-291.

O'Connor, M.I., Piehler, M.F., Leech, D.M., Anton, A. \& Bruno, J.F. (2009). Warming and resource availability shift food web structure and metabolism. PLoS Biol. , 7, e1000178.

Paine, R.T. (1969). A note on trophic complexity and community stability. Am. Nat., 103, 91-93.

Pellissier, L., Albouy, C., Bascompte, J., Farwig, N., Graham, C., Loreau, M., et al. (2018). Comparing species interaction networks along environmental gradients. Biol. Rev. , 93, 785-800.

Pellissier, L., Fiedler, K., Ndribe, C., Dubuis, A., Pradervand, J.N., Guisan, A., et al. (2012a). Shifts in species richness, herbivore specialization, and plant resistance along elevation gradients.Ecol. Evol. , 2, 1818-1825.

Pellissier, L., Rasmann, S., Litsios, G., Fiedler, K., Dubuis, A., Pottier, J., et al. (2012b). High host-plant nitrogen content: A prerequisite for the evolution of ant-caterpillar mutualism? J. Evol. Biol.

Pérez-Harguindeguy, N., Díaz, S., Garnier, E., Lavorel, S., Poorter, H., Jaureguiberry, P., et al. (2013). New handbook for standardised measurement of plant functional traits worldwide. Aust. J. Bot., 61, 167-234.

Petchey, O.L., McPhearson, P.T., Casey, T.M. \& Morin, P.J. (1999). Environmental warming alters food-web structure and ecosystem function. Nature, 402, 69-72.

Pornon, A., Escaravage, N., Burrus, M., Holota, H., Khimoun, A., Mariette, J., et al. (2016). Using metabarcoding to reveal and quantify plant-pollinator interactions. Sci. Rep. , 6, 27282.

Power, M.E., Tilman, D., Estes, J.A., Menge, B.A., Bond, W.J., Mills, L.S., et al. (1996). Challenges in the Quest for Keystones.Bioscience, 46, 609-620.

R Core Team. (2019). R: A language and environment for statistical computing. R Foundation for Statistical Computing, Vienna, Austria. URL https://www.r-project.org/.

Rahbek, C. (1995). The elevational gradient of species richness: a uniform pattern? Ecography (Cop.). , 18, 200-205. 
Rasmann, S., Alvarez, N. \& Pellissier, L. (2014). The altitudinal niche-breadth hypothesis in insect plant interactions. Ann. Plant Rev , 47, 339-360.

Rausher, M.D. (2001). Co-evolution and plant resistance to natural enemies. Nature , 411, 857-864.

Roslin, T., Traugott, M., Jonsson, M., Stone, G.N., Creer, S. \& Symondson, W.O.C. (2019). Introduction: Special issue on species interactions, ecological networks and community dynamics - Untangling the entangled bank using molecular techniques. Mol. Ecol. , 28, 157-164.

Russo, L., Albert, R., Campbell, C. \& Shea, K. (2019). Experimental species introduction shapes network interactions in a plant-pollinator community. Biol. Invasions , 21, 3505-3519.

Sanson, G., Read, J., Aranwela, N., Clissold, F. \& Peeters, P. (2001). Measurement of leaf biomechanical properties in studies of herbivory: Opportunities, problems and procedures. Austral Ecol. , 26, 535-546.

Sentis, A., Hemptinne, J.L. \& Brodeur, J. (2014). Towards a mechanistic understanding of temperature and enrichment effects on species interaction strength, omnivory and food-web structure. Ecol. Lett. , 17, $785-793$.

Thebault, E. \& Fontaine, C. (2010). Stability of ecological communities and the architecture of mutualistic and trophic networks.Science, 329, 853-856.

Thioulouse, J., Dufour, A.B., Jombart, T., Dray, S., Siberchicot, A. \& Pavoine, S. (2018). Multivariate analysis of ecological data with ade4. Multivar. Anal. Ecol. Data with ade4 . Springer New York, New York.

Thompson, R.M., Brose, U., Dunne, J.A., Hall, R.O., Hladyz, S., Kitching, R.L., et al. (2012). Food webs: reconciling the structure and function of biodiversity. Trends Ecol. Evol. , 27, 689-697.

Trøjelsgaard, K. \& Olesen, J.M. (2016). Ecological networks in motion: micro- and macroscopic variability across scales. Funct. Ecol. , 30, 1926-1935.

Tylianakis, J.M., Didham, R.K., Bascompte, J. \& Wardle, D.A. (2008). Global change and species interactions in terrestrial ecosystems.Ecol. Lett., 11, 1351-1363.

Tylianakis, J.M. \& Morris, R.J. (2017). Ecological networks across environmental gradients. Annu. Rev. Ecol. Evol. Syst., 48, annurev-ecolsys-110316-022821.

Unsicker, S.B., Oswald, A., Köhler, G. \& Weisser, W.W. (2008). Complementarity effects through dietary mixing enhance the performance of a generalist insect herbivore. Oecologia , 156, 313-324.

Vázquez, D.P. \& Aizen, M.A. (2003). Null model analyses of specialization in plant-pollinator interactions. Ecology , 84, 2493-2501.

Welti, E., Helzer, C. \& Joern, A. (2017). Impacts of plant diversity on arthropod communities and plantherbivore network architecture.Ecosphere, 8, e01983.

Welti, E.A.R. \& Joern, A. (2015). Structure of trophic and mutualistic networks across broad environmental gradients. Ecol. Evol., 5, 326-334.

Welti, E.A.R., Qiu, F., Tetreault, H.M., Ungerer, M., Blair, J. \& Joern, A. (2019). Fire, grazing and climate shape plant-grasshopper interactions in a tallgrass prairie. Funct. Ecol. , 33, 735-745.

\section{Tables}

Table 1 Coefficients obtained for the observed metric variation along the temperature gradient and the null models. For each metric, the slope of the relationship between the observed metric and the temperature, the intercept estimate, the $p$ value $(P)$, the degrees of freedom $(\mathrm{df})$, the t-value, the standardized effect size (SES) measured between the observed metric slopes and those obtained from random networks of interactions, and the $2.5 \%$ and $97.5 \%$ quantile interval limits of the slopes obtained for random networks are 
given. For generality, weighted nestedness and robustness, observed slopes were found outside the $2.5 \%$ and 97.5\% quantile interval limits obtained with null models.

\begin{tabular}{llllllll}
\hline & Slope Estimate & Intercept Estimate & P & df & t-value & SES & 2.5\% quantil \\
\hline Number of links per species & 0.0819 & 1.44 & 0.003 & 42.56 & 3.20 & -1.82 & 0.0816 \\
Generality & -0.4973 & 36.28 & 0.035 & 43.66 & -2.17 & -3.44 & -0.4066 \\
Weighted nestedness & -0.0102 & 0.40 & 0.042 & 43.91 & -2.10 & -3.51 & -0.0054 \\
Robustness & 0.0033 & 0.74 & 0.023 & 43.62 & 2.06 & -5.77 & 0.0057 \\
\hline
\end{tabular}

\section{Figures}

Figure 1 Plant-orthoptera ecological networks for low- and high-elevation sites situated along three elevation transects. For each network, yellow bars represent species of orthoptera and green bars species of plant. To ease graph interpretation, species labels are grouped into genera for orthoptera and into families for plants, sorted according to the phylogeny. For species belonging to non-monophyletic genera (e.g. Chorthippus ), taxa are indicated with a number following the genus name. The reconstruction of plant and insect phylogenies is detailed in Appendix S1, section Methods. Bars with no label correspond to the taxa of the preceding labelled bar, reading from left to right. The width of the links represents interaction intensity.

Figure 2 Relationship between the mean summer temperature at each site along the elevation gradients and various plant-orthoptera network metrics: number of links per species (a), network generality (b), weighted nestedness (c), and network robustness (d). Expected metrics based on null models are represented in light gray. Regression lines result from linear mixed effects models, where solid lines indicate a significant relationship between the observed or random network metrics and the temperature. For empirical networks, the confidence interval and regression line of the temperature vs.metric relationship is shown. When the slope of the empirical relationship is outside the 2.5-97.5\% quantile interval of the slopes obtained from random networks, the regression line and metric values are red, while they are dark grey when the observed slope is not outside the slope interval expected from null models.

Figure 3 Distribution of keystone species in the functional space of the plant traits between low- (a, $<1050$ $\mathrm{m}$ a.s.l.) and high-elevation (b, $>2000 \mathrm{~m}$ ) plant communities. Plant species are projected onto the first two axes of the principal components analysis (PCA) performed on plant functional traits, which explain $58.8 \%$ (PC1) and 15.6\% (PC2) of the variance. The top 10 keystone species are colored by plant taxonomic group, while all other species are shown in black. The keystone score corresponds to the mean number of secondary extinctions caused by the removal of the plant species. It varies between 0.12 and 0.75 secondary extinctions across all networks but is summarized in the legend as three circle sizes (low, medium and high). Correlations between plant functional traits $(\mathrm{C} / \mathrm{N}, \mathrm{LDMC}$, punch and SLA) and the two first axes of the $\mathrm{PCA}$ are given in the plot in the top right corner.

Figure 4 Matrix representation of the plant-orthoptera ecological networks for pairs of low- and highelevation sites found along three elevation transects. Columns and rows correspond respectively to orthopteran and plant species. Interaction intensity is indicated by the color gradient with dark blue corresponding to maximal interaction strength and white to interaction absence. 
Low elevation
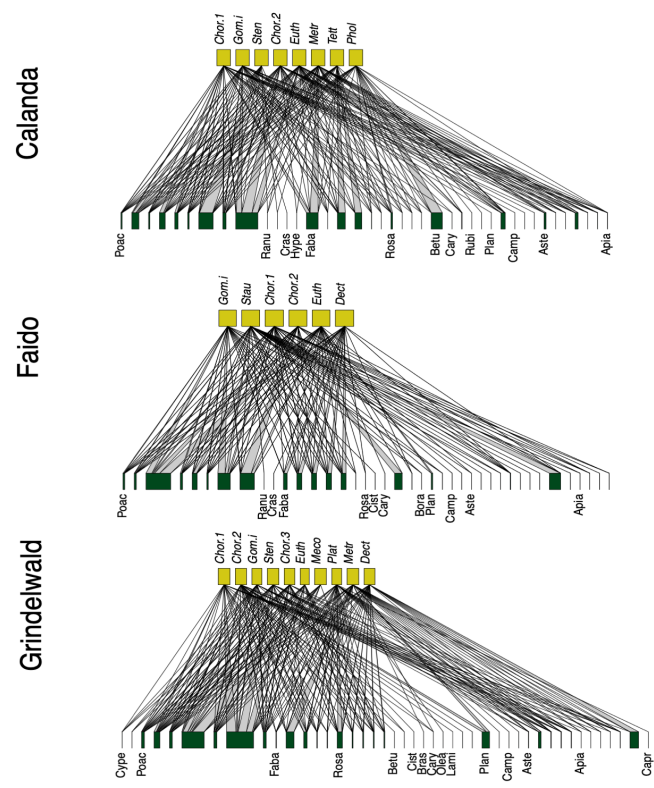

High elevation
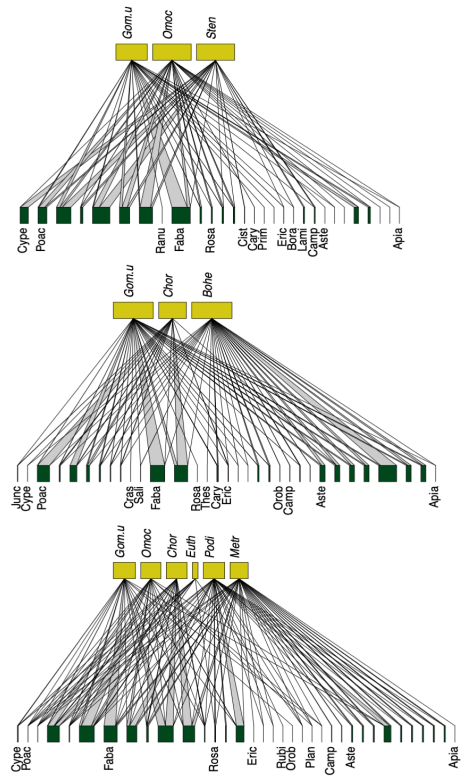
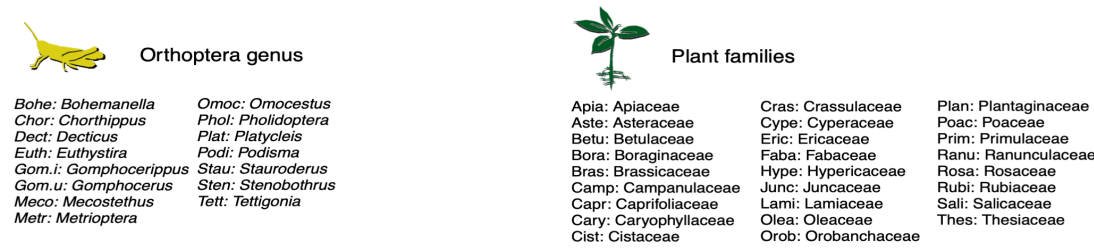

Fig. 1

\section{Hosted file}

image2.emf available at https://authorea.com/users/317969/articles/447994-the-structure-ofplant-herbivore-ecological-networks-varies-along-elevation-gradients

Fig. 2

\section{Hosted file}

image3.emf available at https://authorea.com/users/317969/articles/447994-the-structure-ofplant-herbivore-ecological-networks-varies-along-elevation-gradients

Fig. 3

\section{Hosted file}

image4.emf available at https://authorea.com/users/317969/articles/447994-the-structure-ofplant-herbivore-ecological-networks-varies-along-elevation-gradients

Fig. 4 
Low elevation
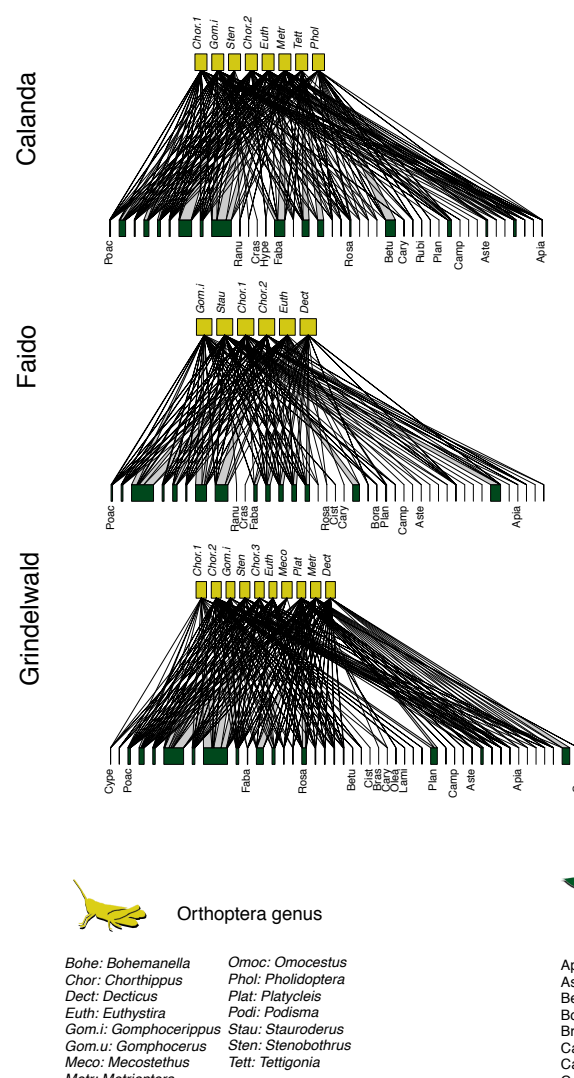

High elevation
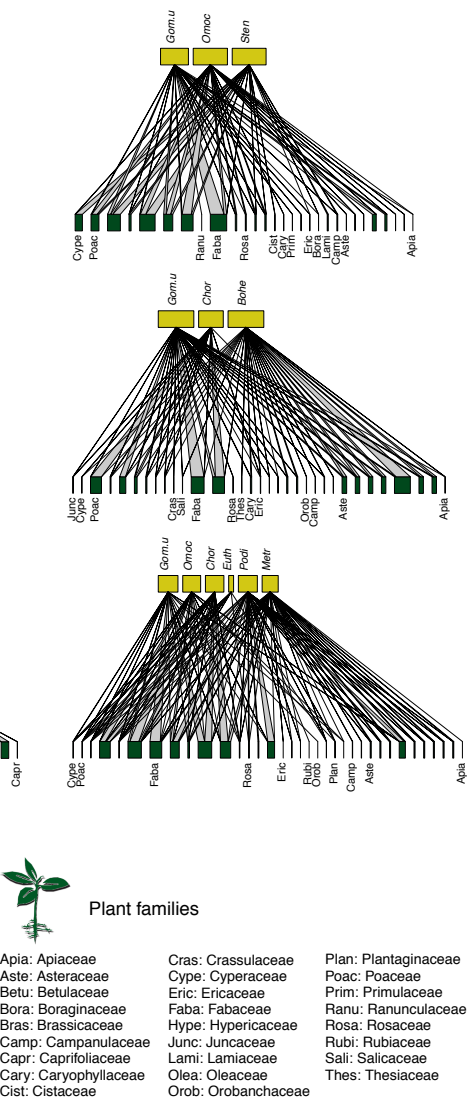

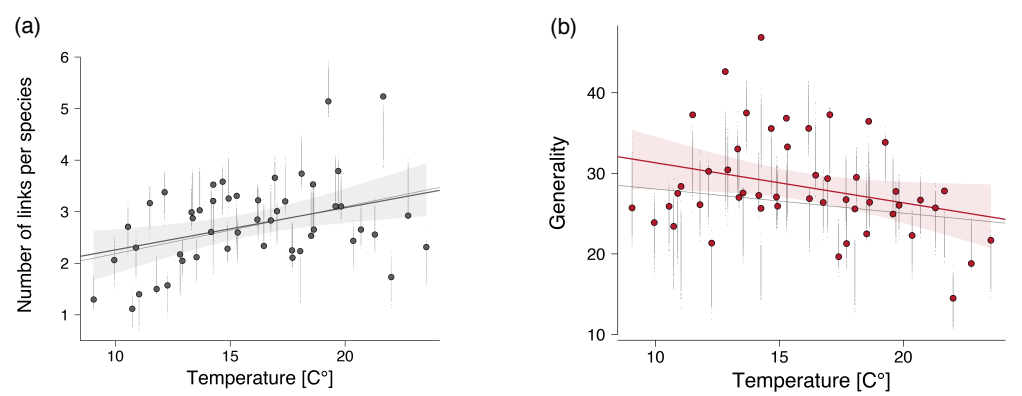

(c)

(d)
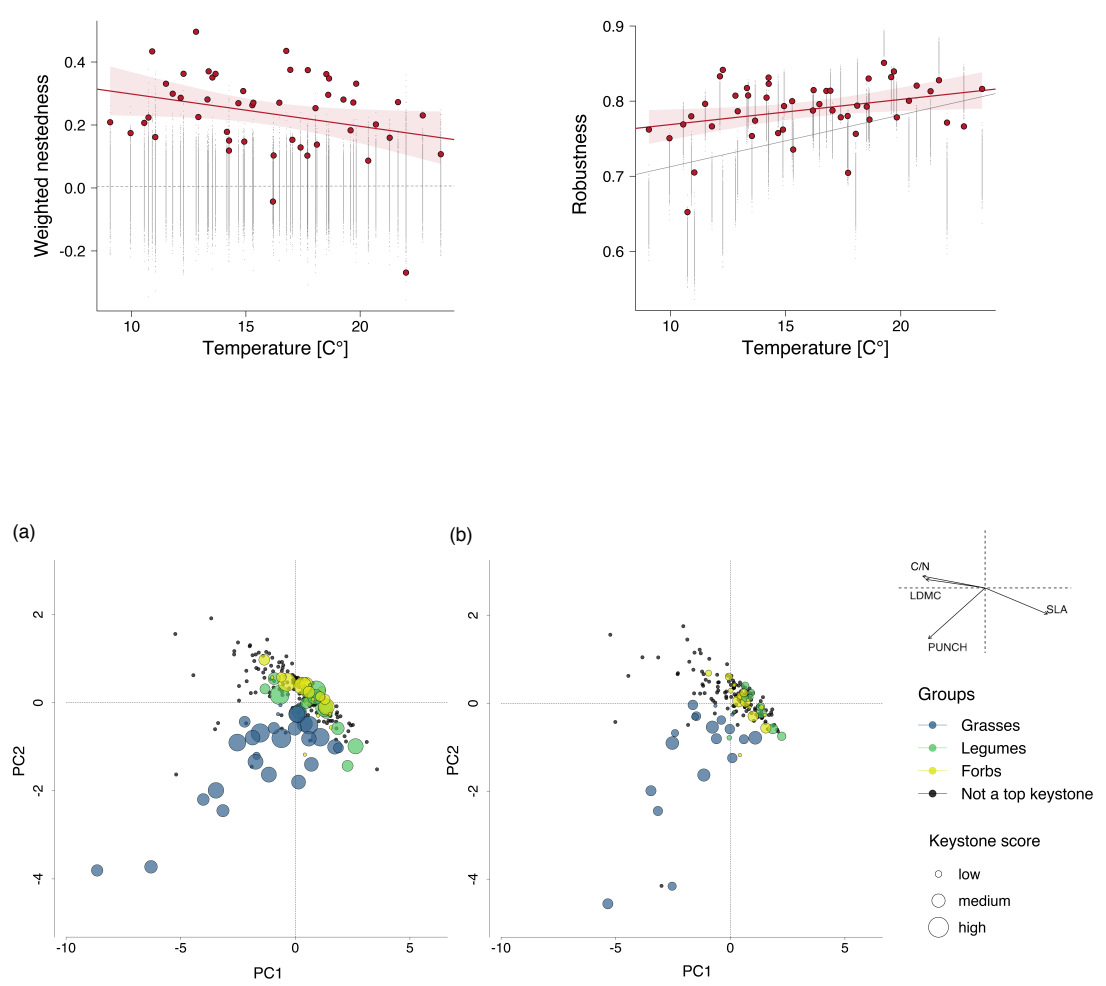


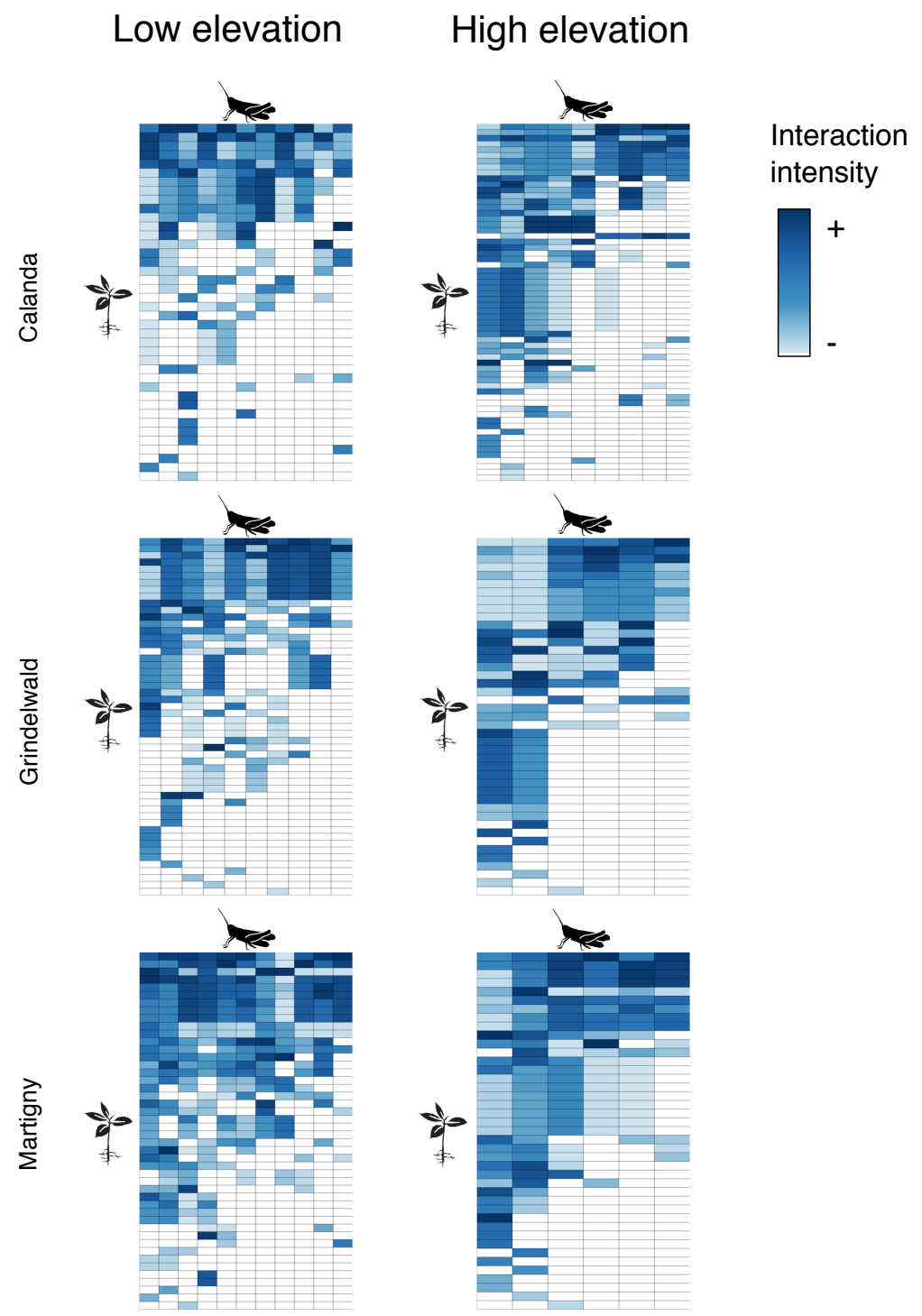

